\title{
Biological pretreatment of lignocellulosic biomass for volatile fatty acid production
}

\begin{abstract}
Volatile fatty acid (VFA) platform has been suggested as a potential alternative to sugar platform for the production of biofuels and fine chemicals. Biomass-derived VFA is one of the potential alternatives for VFA production that required only a mild pretreatment process to open up the lignocellulosic biomass structure for anaerobic digestion (AD), whereas some of the biomass like food waste, manure, sludge, or any biodegradable biomass do not require any pretreatments. Lignocellulosic biomass such as hardwood (forestry and agricultural biomass) does require pretreatments to remove lignin or to alter the lignocellulosic compositions. Besides, VFA also can be produced from all types of organic polymers (carbohydrates, protein, and lipid) that will increase the VFA yield as compared with sugar platform, which can be produced from carbohydrate polymers only. Biological pretreatment of lignocellulosic biomass offers an advantage for the production of VFA. This chapter will focus on suitable pretreatment strategies for VFA production especially on the biological pretreatments.
\end{abstract}

